\title{
Pruning the projections
}

\section{ce \\ activation of \\ frontal cortex \\ neurons that \\ project to \\ the VTA/SNc \\ in wild-type \\ mice induced \\ locomotor \\ hyperactivity \\ and increased \\ dopamine \\ release in the \\ striatum}

Psychiatric disorders are associated with abnormalities in neuronal structures such as dendritic spines and with widespread disruption in neural circuits, but it is not yet clear how these different disease processes interact. Kim et al. now show that disruption of actin dynamics in the frontal cortex of mice, which results in abnormal dendritic spine morphology, leads to long-range circuit disruptions and psychomotor dysfunction, a phenotype related to positive symptoms of psychosis in humans.

Actin-related protein 2/3 complex subunit 3 (ARPC3) is required for actin polymerization in spines. In mice, conditional knockout of Arpc3 selectively in excitatory cortical neurons resulted in progressive loss

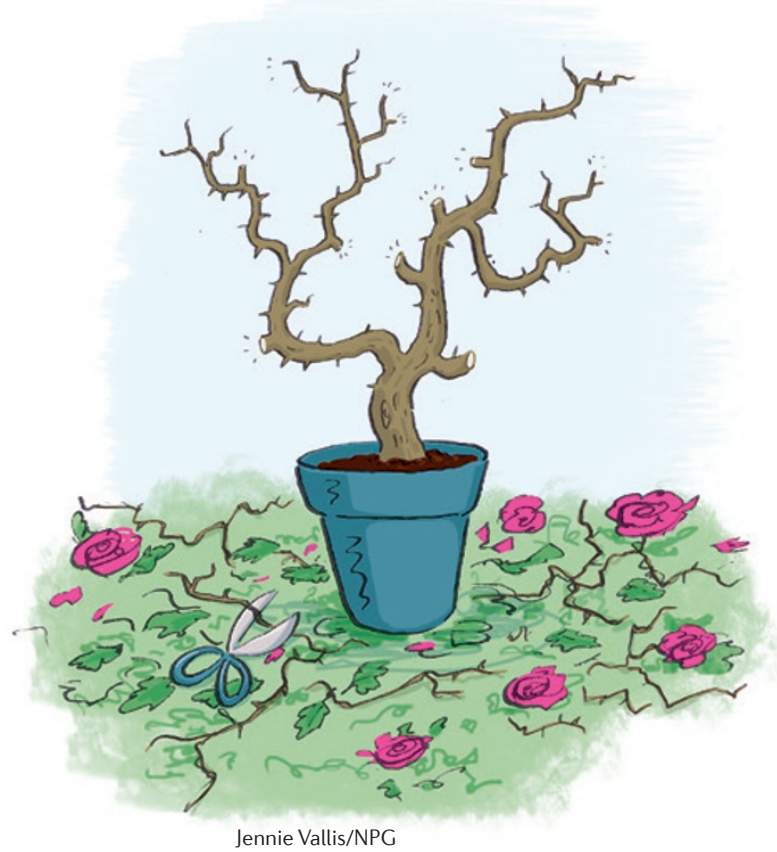

of dendritic spines. Furthermore, these mice developed locomotor hyperactivity that was reversed by antipsychotic drugs, which are thought to mediate their effects by blocking dopamine signalling in the striatum.

Arpc3-mutant mice also had increased levels of dopamine and its metabolites in the ventral striatum, suggesting that the behavioural abnormalities could be due to increased dopaminergic signalling in this region. Notably, however, dendritic spines on striatal neurons, where Arpc3 expression was intact, were not disrupted. This suggested that, although the behavioural phenotype was associated with striatal dysfunction, the primary neurobiological deficit responsible for the phenotype was located elsewhere in the circuit.

To identify which brain area was responsible, localized re-expression of $A r p c 3$ was carried out in the mutant mice. Re-expression of $A r p c 3$ in the excitatory neurons in the frontal cortex, but not in other forebrain regions, such as the hippocampus, corrected locomotor hyperactivity and decreased striatal dopamine levels, suggesting that disruption of actin dynamics in frontal cortex neurons triggers dysregulation of the psychomotor circuit.

The authors next investigated whether the ventral tegmental area and substantia nigra pars compacta (VTA/SNc), a major source of dopaminergic input to the striatum, were also part of the locomotor hyperactivity circuit that was disrupted in the Arpc3-mutant animals. Anterograde tracing in these mice showed that frontal cortex neurons project to the VTA/SNc.

However, it was unclear how increased dopamine release in the striatum occurred in response to spine loss in the frontal cortex, as loss of dendritic spines would typically be expected to reduce neuronal excitability. Interestingly, electron microscopy showed that Arpc3mutant animals had an increase in the number of abnormal synapses in the frontal cortex, and whole-cell patch clamp recordings of frontal cortex neurons projecting to the VTA/SNc indicated that this was associated with increased neuronal excitation. In addition, optogenetic activation of frontal cortex neurons that project to the VTA/SNc in wildtype mice induced locomotor hyperactivity and increased dopamine release in the striatum, confirming the functional importance of this circuit.

Together, these findings show that disruption of actin cytoskeletal dynamics and dendritic spine morphology can result, paradoxically, in an increase in neuronal excitability, and reveal a frontal cortex-midbrain circuit underlying locomotor hyperactivity, which might help to explain how abnormal behaviours arise in psychiatric disease.

Fiona Carr

ORIGINAL RESEARCH PAPER Kim, I. H. et al. Spine pruning drives antipsychotic-sensitive locomotion via circuit control of striatal dopamine.

Nat. Neurosci. http://dx.doi.org/10.1038/nn.4015 (2015) 